\title{
EDITORIAL
}

\section{MAKING DOUble PATTERNING COST SINGLE}

The only lithography technology that is readily available and has a full infrastructure is double patterning with ArF immersion scanners. Single patterning is being used for applications with $\mathrm{k}_{\mathrm{l}}$ between 0.26 and 0.3 . In principle, double patterning can reduce $k_{1}$ by half. This means memory products at half pitches below $19 \mathrm{~nm}$ and at least I5-nm nodes for logic products. Unfortunately, double patterning is costly. Not only are exposure and mask costs doubled, the cost for pattern transfer, such as etching, is also doubled. In addition, the circuit patterns need additional restrictions to confine the splitting to only two masks. If left unrestricted, three or even four masks may be needed, further escalating the cost. Needless to say, the overlay accuracy between exposures adds an extra requirement to the exposure tool.

There might be a way to make the cost of double patterning comparable to single patterning. I can visualize two fronts of improvement: namely, make double exposure cost mimic single exposure, and double patterning cost approach that of double exposure. Putting the two together can result in very reasonable cost, thus removing a very important obstacle to using double patterning.

The conventional scanner can be equipped with two illumination trains with a more powerful laser split into two beams. Two masks on an extended reticle stage can be exposed simultaneously and the beams can be recombined with a polarized beamsplitter and exposed simultaneously on the wafer. Alternately, they can be brought to close vicinity with two bending mirrors and a reflecting wedge to be exposed in quick succession during the scan- ning process. The scanner allows a much more compact setup than steppers. The cost of a more powerful laser, extra illumination train, larger reticle stage, and faster track is only a fraction of the cost of two independent scanners and two tracks with equivalent throughput. An additional benefit is that the masks can be prealigned with respect to each other, eliminating the contribution from overlay tolerance between the two masks.

The problem with double exposure without freezing or repatterning is that the resist tends to accumulate the background exposure from the first exposure, resulting in superposition of residual exposures at the areas that are supposed to be unexposed. One only has to develop a resist whose sensitivity to below-threshold exposure is half of the resist for single exposure to make the double exposure accumulation of residual exposure equal to that of single exposure.

Hence, combining a twinilluminator scanner and a lowresidual-exposure resist can make the cost of double patterning approach that of single patterning.

Happy reading!

Burn J. Lin

Editor-in-Chief

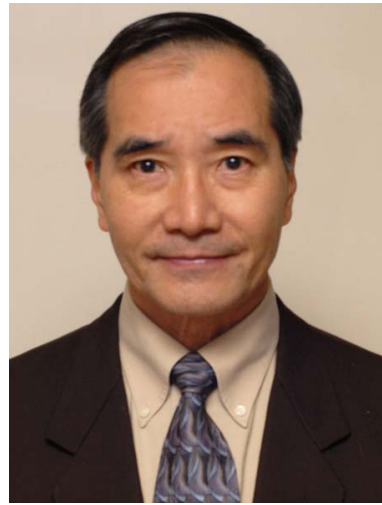

